\title{
A newly developed anti-Mucin 13 monoclonal antibody targets pancreatic ductal adenocarcinoma cells
}

\author{
YUKARI NISHII $^{1}$, MIKI YAMAGUCHI ${ }^{1}$, YASUTOSHI KIMURA ${ }^{2}$, TADASHI HASEGAWA $^{3}$, \\ HIROYUKI ABURATANI ${ }^{4}$, HIROAKI UCHIDA ${ }^{5}$, KOICHI HIRATA ${ }^{2}$ and YUJI SAKUMA ${ }^{1}$
}

\begin{abstract}
Departments of ${ }^{1}$ Molecular Medicine, Research Institute for Frontier Medicine, ${ }^{2}$ Surgery, Surgical Oncology and Science and ${ }^{3}$ Surgical Pathology, Sapporo Medical University School of Medicine, Sapporo; ${ }^{4}$ Genome Science Division,

Research Center for Advanced Science and Technology, The University of Tokyo; ${ }^{5}$ Laboratory of Oncology,

Department of Life Sciences, Tokyo University of Pharmacy and Life Sciences, Tokyo, Japan
\end{abstract}

Received November 21, 2014; Accepted January 5, 2015

DOI: $10.3892 / \mathrm{ijo} .2015 .2880$

\begin{abstract}
Pancreatic cancer is one of the most severe forms of malignancy. Patients with unresectable or metastatic pancreatic cancer usually receive chemotherapy that causes various adverse effects. Antibody-drug conjugates (ADCs), drugs developed by conjugating an anticancer agent to a monoclonal antibody (mAb), can alleviate the side effects of chemotherapy because ADCs selectively bind to cancer cells expressing a particular antigen. We recently developed the recombinant protein DT3C comprising diphtheria toxin (DT) lacking the receptor-binding domain but containing the C1, C2, and C3 domains of Streptococcus protein G (3C). The $\mathrm{mAb}-\mathrm{DT} 3 \mathrm{C}$ conjugates can be used to select $\mathrm{mAbs}$ that are internalized by cells, because the conjugates decrease cell viability only when they are internalized by cells through $\mathrm{Ab}$-antigen reactions. We developed a new $\mathrm{mAb}$ to be internalized by TCC-PAN2 cells, a pancreatic carcinoma cell line. The $\mathrm{mAb}$, designated TCC56, recognized Mucin 13 (MUC13), while TCC56-DT3C conjugates induced cell death in TCC-PAN2 cells expressing MUC13. We found that MUC13 was expressed, at least partially, in all 40 pancreatic ductal carcinoma tissues and adjacent non-cancerous tissues analyzed. The expression levels of MUC13 in pancreatic cancer tissues were greater than those in normal tissues. Our findings suggest that MUC13 can be a target molecule for pancreatic cancer treatment. ADCs, including mAb TCC56, could be promising anticancer agents to alleviate the adverse effects of chemotherapy.
\end{abstract}

Correspondence to: Dr Yuji Sakuma, Department of Molecular Medicine, Research Institute for Frontier Medicine, Sapporo Medical University School of Medicine, South 1, West 17, Chuo-ku, Sapporo 060-8556, Japan

E-mail: sakuma@sapmed.ac.jp

Key words: pancreatic ductal carcinoma, mucin 13, monoclonal antibody, antibody-drug conjugate, internalization, immunohistochemistry

\section{Introduction}

Pancreatic cancer, of which $\sim 85 \%$ are ductal adenocarcinomas, remains one of the most aggressive and deadly malignancies worldwide (1). In the USA, pancreatic cancer is diagnosed in $\sim 46,000$ people annually, with 40,000 of these patients dying (2). Surgical resection is a potential curative therapy for pancreatic cancer; however, $\sim 80 \%$ of patients are diagnosed too late to be operated on because of vague symptoms and lack of an efficient screening method to detect pancreatic cancer at an earlier stage (1). Even patients who have undergone resection have a poor prognosis because pancreatic cancers often relapse. Patients with unresectable or metastatic pancreatic cancer usually receive chemotherapy with gemcitabine alone, or are subject to combination chemotherapy such as gemcitabine plus albumin-bound paclitaxel particles, or a FOLFIRINOX regimen (oxaliplatin, irinotecan, fluorouracil and leucovorin) (3-5). Although the efficacy of the FOLFIRINOX regimen in patients results in considerable improvement, it often causes adverse side effects such as fatigue, neutropenia, anemia, vomiting, diarrhea, and sensory neuropathy $(4,6)$. These adverse effects occur because anticancer drugs affect cancer cells and normal cells. There is an urgent need for the development of drugs that can selectively target and kill malignant cells without adversely affecting patients. Antibody-drug conjugates (ADCs) are drugs developed through the conjugation of an anticancer agent to a monoclonal antibody (mAb), thereby making them selective for certain cells (7). Antibodies for ADCs must be internalized by the target cells, but few methods are available for screening the ability of mAbs to be internalized by cells. We recently developed a recombinant protein, DT3C, comprising diphtheria toxin (DT) lacking the receptor-binding domain but containing the $\mathrm{C} 1, \mathrm{C} 2$, and C3 domains of Streptococcus protein G (3C) (8). When a $\mathrm{mAb}-\mathrm{DT} 3 \mathrm{C}$ conjugate, which functions like an ADC in vitro, reduces the viability of cancer cells, the $\mathrm{mAb}$ being tested must have been internalized by target cells. This screening method using DT3C has enabled us to develop a new $\mathrm{mAb}$ that recognizes mucin 13 (MUC13), and is internalized by pancreatic cancer cells. 
Histologically, most pancreatic cancers are ductal adenocarcinomas. Glandular epithelia, from which adenocarcinoma cells arise, produce mucin, a macromolecule glycoprotein. The mucin family of proteins covers the apical surface of the respiratory, gastrointestinal, or genital tract; they can be categorized as secreted or transmembrane type proteins $(9,10)$. It appears that transmembrane mucins, whose expression is often increased in cancer cells, play an important role in the development or maintenance of adenocarcinomas (9-12). MUC13, our focus in the current study, is a transmembrane mucin and is expressed at the mRNA and protein levels in several normal glandular epithelia such as the intestine, colon or pancreatic duct (13). Expression levels of MUC13 appear to be upregulated in gastric, colonic, pancreatic, and ovarian cancers. Increased expression of MUC13 seems to enhance the proliferation, migration, or invasion of cancer cells (14-21). To the best of our knowledge, no reports have shown that MUC13 is a candidate target molecule that ADCs can bind to. Additionally, there are only two reports that reveal the expression of MUC13 in pancreatic cancers $(18,20)$. Our objective was to determine if MUC13 could be a target molecule in pancreatic cancer therapy, and we have found that pancreatic carcinoma tissues examined expressed more MUC13 than that in normal tissues, and that anti-MUC13 mAb-DT3C conjugates induced cell death in pancreatic cancer cells.

\section{Materials and methods}

Ethics. The experimental procedures were approved by the Institutional Review Board at Sapporo Medical University.

Cell lines. We used the human TCC-PAN2 pancreatic cancer cell line (Japanese Collection of Research Bioresources Cell Bank, Osaka, Japan), the mouse myeloma P3U1 cell line (Japanese Collection of Research Bioresources Cell Bank), and the CHO-K1 Chinese hamster ovary cell line (American Type Culture Collection, Manassas, VA, USA). All cell lines were cultured in RPMI-1640 or DMEM supplemented with $10 \%$ (v/v) Super Low IgG-FBS (Hyclone, Thermo Scientific, Waltham, MA, USA), $1 \mathrm{mM}$ sodium pyruvate (Life Technologies Japan, Tokyo, Japan), and $1 \%$ (v/v) streptomycin-penicillin-glutamine solution (Life Technologies) at $37^{\circ} \mathrm{C} / 5 \% \mathrm{CO}_{2}$.

Production of DT3C. DT3C was produced and purified as described previously (8).

Production and screening of hybridomas. We intraperitoneally injected a Balb/c mouse with phosphate-buffered saline (PBS) containing $5 \times 10^{6}$ TCC-PAN2 cells every 7 days. A final booster injection was administered after 7 days with the same number of the cells (Fig. 1A). Two days after the final injection the mouse was sacrificed, and $7.75 \times 10^{7}$ splenocytes were fused with $1.55 \times 10^{7} \mathrm{P} 3 \mathrm{U} 1$ cells using polyethylene glycol. When the hybridomas had grown to $\sim 50 \%$ confluence, the culture supernatant fluid that was likely to contain polyclonal antibodies was tested for antibody internalization. The supernatant from each well, and DT3C, were incubated in 96-well microplates at room temperature for $30 \mathrm{~min}$ to form Ab-DT3C conjugates. TCC-PAN2 cells were then added to each well at a concentration of $1 \times 10^{4}$ cells/well and incubated for $72 \mathrm{~h}$ to evaluate the degree of survival for TCC-PAN2 cells in each well in the presence of Ab-DT3C conjugates. The number of viable cells after treatment was then estimated using a WST-1 assay (Roche Diagnostics, Indianapolis, IN, USA). The hybridomas that induced extensive cell death were selected and cloned by limiting dilution. The mouse $\mathrm{mAb}$ was purified using Protein G Sepharose 4 Fast Flow (GE Healthcare Japan, Tokyo, Japan). A Mouse Monoclonal Antibody Isotyping kit (Roche Applied Science, Mannheim, Germany) was used to identify the antibody isotype.

Immunoprecipitation and mass spectrometry. We prepared TCC-PAN2 cells and biotinylated their surfaces using a Biotinylation kit (Thermo Scientific). Membranes were solubilized on ice for $30 \mathrm{~min}$ in $1 \mathrm{ml}$ of buffer comprising $1 \%(\mathrm{v} / \mathrm{v})$ $\mathrm{NP} 40,50 \mathrm{mM}$ Tris- $\mathrm{HCl} \mathrm{pH} 7.6,150 \mathrm{mM} \mathrm{NaCl}$, and a protease inhibitor cocktail (Roche Applied Science). A mouse mAb, TCC56, which we identified and was extensively analyzed in this study, and a control mouse IgG3 (BD Biosciences, Tokyo, Japan) were used. Details of these experiments have been described previously $(22,23)$.

Confirmation of mass spectrometry results. MUC13 or control cDNA were ligated into the pEZ-M02 expression vector (GeneCopoeia, Rockville, MD, USA). The recombinant plasmid was transfected into CHO-K1 cells using FuGENE HD Transfection reagent (Promega KK, Tokyo, Japan). At $48 \mathrm{~h}$ post-transfection, transfected cells were trypsinized, washed and suspended in staining buffer (2\% FBS/PBS) containing saturating amounts of anti-MUC13 (TCC56) or negative control mouse IgG1 (clone P3; eBioScience, Affymetrix Japan, Tokyo, Japan). The reactivity of each $\mathrm{mAb}$ was analyzed by flow cytometry using a FACSCalibur (BD Biosciences).

Evaluating mAb internalization by cells. A mAb and DT3C were incubated at room temperature for $30 \mathrm{~min}$ to generate the mAb-DT3C conjugate. TCC-PAN2 cells were seeded and incubated in the presence of the mAb-DT3C conjugate for $120 \mathrm{~h}$. The viability of cancer cells was assessed using a WST-1 assay. We determined, and used a quantity of $\mathrm{mAb}$ required for sufficient formation of $\mathrm{mAb}$-DT3C conjugates at each DT3C concentration tested. In theory, each conjugate consisted of one mAb molecule $(150 \mathrm{kDa})$ and two DT3Cs (140 kDa) (8).

Primary pancreatic cancer tissues. Patients $(\mathrm{n}=156)$ were operated on for pancreatic ductal cancer at Sapporo Medical University Hospital between August 2001 and February 2013. We selected 40 patients whose tumors were resected completely (R0 resection) and who were followed up after surgery on a regular basis. The patient group comprised 22 men and 18 women, with a median age of 69 years (range, $41-84$ years). All tumor slides were stained with hematoxylin and eosin (HE), and reviewed by one of the authors (Yuji Sakuma) to verify diagnosis. We classified 35 tumors as well differentiated $(n=17)$, moderately differentiated $(n=14)$, and poorly differentiated $(n=4)$ carcinomas, and designated these as invasive ductal adenocarcinomas. The other tumors $(n=5)$ were adenosquamous cell carcinomas according to histological results. Pathological examination confirmed that all tumors 
A

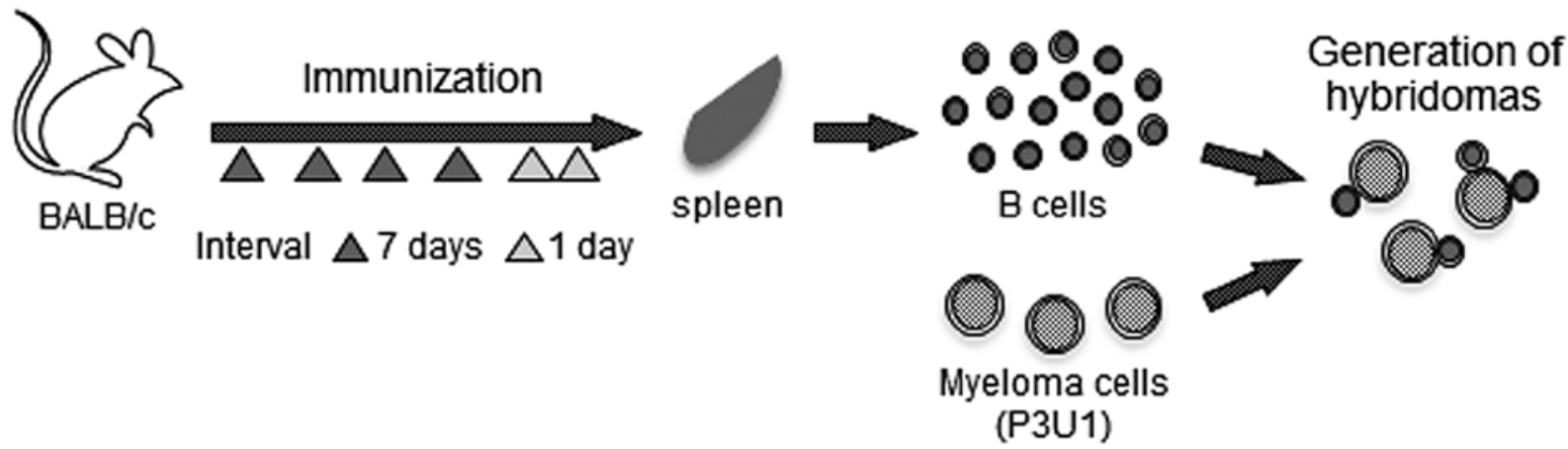

\section{HAT selection}
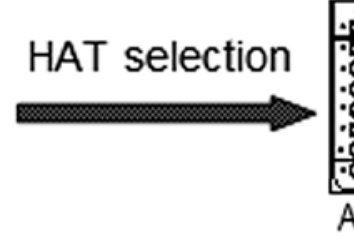

\section{Screen Limiting dilution}

B

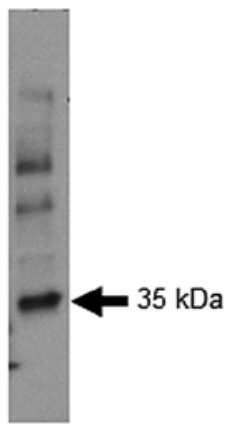

D
CHO-K1/MOCK (pEZ-M02)

CHO-K1/h-MUC13 (pEZ-M02 MUC13)

C $35 \mathrm{kDa}$

\begin{tabular}{|c|c|c|c|c|c|c|c|}
\hline $\mathrm{N}$ & Unused & Total & $\%$ Cov & $\% \operatorname{Cov}(50)$ & $\% \operatorname{Cov}(95)$ & Name & Peptides $(95 \%)$ \\
\hline $\mathbf{1}$ & $\mathbf{1 2 . 1 8}$ & $\mathbf{1 2 . 2}$ & $\mathbf{1 6 . 6 0 0 0}$ & $\mathbf{1 3 . 4 8 0 0}$ & $\mathbf{1 3 . 4 8 0 0}$ & Mucin $\mathbf{1 3}$ & $\mathbf{8}$ \\
\hline
\end{tabular}

\section{Amino acid sequence of MUC13}

1 mkaiihltll allsvntatn ggnsadavtt tetatsgptv aaadtetnf petasttant

61 psfptatspa ppiisthsss tiptpappii sthssstipi ptaadsestt nvnslatsdi 121 itasspndgl itmvpsetqs nnemsptted nqssgpptgt alletstlns tgpsnpcqdd 181 pcadnslcvk lhntsfclcl egyyynsstc kkgkvfpgki svtvsetfdp eekhsmayqd 241 lhseitslfk dvfgtsvygq tviltvstsl sprsemradd kfvnvtivti laettsdnek 301 tvtekinkai rssssnflny dltlrcdyyg cnqtaddcln glacdcksdl qrpnpqspfc 361 vasslkcpda cnaqhkqcli kksggapeca cvpgyqedan gncqkcafgy sgldckdkfq 421 liltivgtia givilsmiia livtarsnnk tkhieeenli dedfqnlklr stgftnlgae 481 gsvfpkvit asrdsqmqnp ysrhssmprp dy
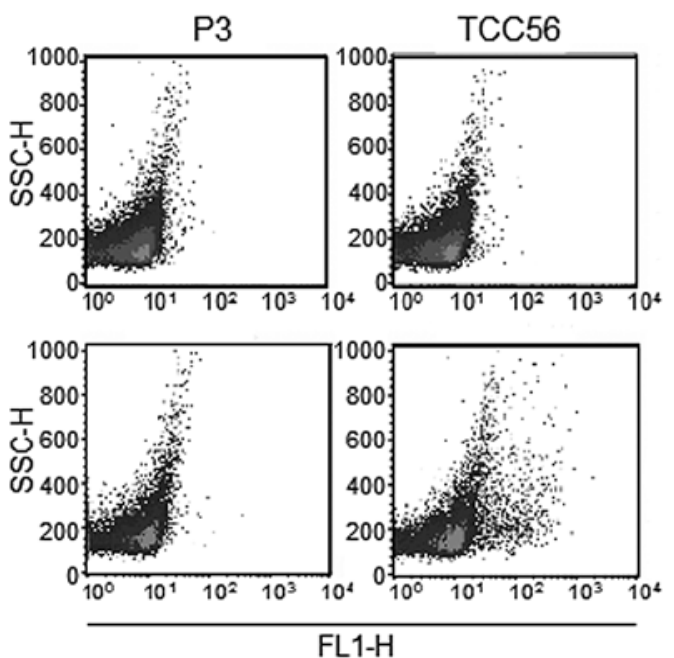

Figure 1. Identification of the mAb TCC56 antigen. (A) Schematic for HAT (hypoxanthine-aminopterin-thymidine) selection that was conducted to select hybridomas. (B) Lysates of TCC-PAN2 cells were immunoprecipitated with mAb TCC56. The band at $35 \mathrm{kDa}$ clearly appears under reducing conditions. (C) Identification of the $35 \mathrm{kDa}$ band as MUC13 using mass spectrometry. Boldface type indicates the sequence of the detected peptides. (D) Flow cytometry results indicate the reactivity of $\mathrm{mAb}$ TCC56 or $\mathrm{P} 3$ (a negative control mouse IgG1) with CHO-K1 cells transfected with a MUC13 expression vector. The mAb TCC56 reacted with transfectants expressing MUC13.

were completely resected, 25 of the 40 patients died within two years after surgery, while the remaining patients $(\mathrm{n}=15)$ survived for three years or more without evidence of recurrence after the operation.
MUC13 immunohistochemistry. Immunohistochemical staining for MUC13 was performed on formalin-fixed, paraffin-embedded (FFPE) pancreatic cancer tissue sections. We selected a representative FFPE tissue block from each 
A

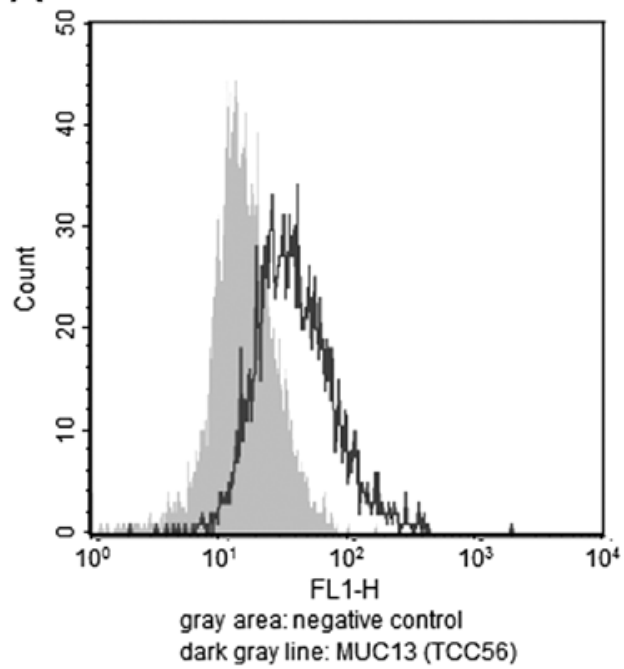

B

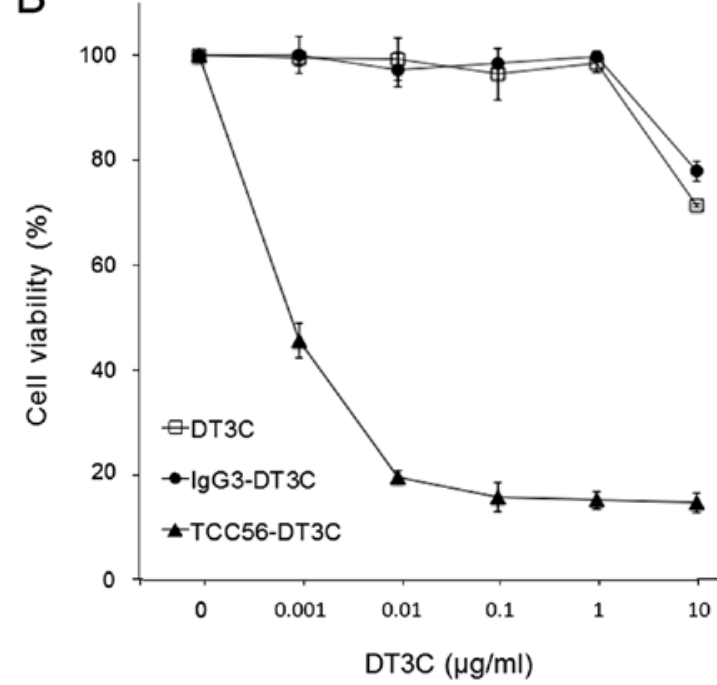

Figure 2. mAb TCC56-DT3C conjugate induced TCC-PAN2 cell death. (A) Flow cytometry results show the reactivity of mAb TCC56 with TCC-PAN 2 cells. MUC13 expression in TCC-PAN2 cells was confirmed. (B) TCC-PAN2 cells were seeded and incubated with DT3C, IgG3-DT3C conjugate, or TCC56-DT3C conjugate. The number of viable cells was evaluated using WST-1 assays. Values are presented as means \pm SD from triplicate cultures.

patient that contained non-cancerous and cancerous pancreatic tissue to precisely evaluate MUC13 expression in both regions. Whole tissue sections were retrieved using Novocastra Epitope Retrieval Solutions pH 6.0 (Leica Biosystems, Nussloch, Germany) at $100^{\circ} \mathrm{C}$ for $20 \mathrm{~min}$. An anti-MUC13 mAb (clone ppz0020), which was developed previously (14), was used as a primary antibody at $10 \mu \mathrm{g} / \mathrm{ml}$, and immunohistochemical staining conducted using a Leica BOND-MAX (Leica). Immunohistochemical staining of normal pancreatic ducts was used as an internal positive control. MUC13 membrane staining was scored based on intensity $(0$, none; 1 , weak; 2 , intermediate; 3 , strong) and proportion of carcinoma or normal ductal cells expressing the molecule $(0$, none; $1,<1 / 100 ; 2,1 / 100-1 / 10 ; 3,1 / 10-1 / 3 ; 4,1 / 3-2 / 3 ; 5,>2 / 3)$ using the Allred scoring method (24).

Statistical analysis. We used the Wilcoxon signed-ranks test to evaluate the differences in Allred scores for MUC13 expression between cancerous and non-cancerous ductal cells. Differences in Allred score among the four tumor subtypes were evaluated by the Kruskal-Wallis test. We used the KaplanMeier method to estimate overall survival; the survival curves between the high expression (Allred score $\geq 6$ ) and low expression ( $\leq 5)$ groups of patients were compared using the log-rank test. A P-value $<0.05$ was considered significant. All statistical calculations were performed with JMP software (JMP for Windows version 7; SAS Institute Japan; Tokyo, Japan).

\section{Results}

Establishment of anti-MUC13 mAb TCC56. We cloned hybridomas from wells where Abs contained in each well and DT3C conjugate (Ab-DT3C conjugates) had substantially decreased viability of TCC-PAN2 cells. Cell viability in each well reflected the efficiency of internalization for Ab-DT3C conjugates into TCC-PAN2 cells. We consequently established a hybridoma secreting mAb TCC56 (Fig. 1A), which was an
$\operatorname{IgG} 3 \kappa$ isotype. Biotinylated proteins were at $35 \mathrm{kDa}$ by immunoprecipitation using mAb TCC56 under reducing conditions (Fig. 1B). MUC13 was identified as a possible candidate of the $35 \mathrm{kDa}$ molecule by mass spectrometry (Fig. 1C). Flow cytometry results indicated that $\mathrm{mAb}$ TCC56 reacted with transfectants expressing MUC13 (Fig. 1D).

The mAb TCC56-DT3C conjugate kills TCC-PAN2 cells. We confirmed that the MUC13 protein was expressed in TCC-PAN2 cells by flow cytometry (Fig. 2A). We assessed to what degree the TCC56-DT3C conjugate could induce cell death in TCC-PAN2 cells. The TCC56-DT3C conjugate decreased the viability of TCC-PAN2 cells in a concentrationdependent manner; viability was $<20 \%$ at $0.1 \mu \mathrm{g} / \mathrm{ml}$ DT3C, while neither DT3C alone nor an IgG3-DT3C (control) conjugate was able to induce cell death even at $1 \mu \mathrm{g} / \mathrm{ml}$ DT3C (Fig. 2B).

Pancreatic ductal adenocarcinoma cells express higher levels of MUC13. In non-cancerous pancreatic tissues, MUC13 expression was observed in ductal cells and some islet cells. While the protein was localized at the apical membrane of ductal cells, islet cells positive for MUC13 staining showed it was present throughout the cytoplasm, but was not observed at the membrane (Fig. 3A), suggesting that the expression observed in islet cells was due to non-specific staining. In contrast, of all 40 cancer tissues examined MUC13 was expressed in varying degrees and was confined to the apical membrane of glands where adenocarcinoma cells formed (Fig. 3A). Expression levels of MUC13 varied substantially among histological subtypes $(\mathrm{p}=0.0122)$; well or moderately differentiated ductal adenocarcinomas expressed MUC13 at higher levels than in poorly differentiated ductal adenocarcinomas or adenosquamous cell carcinomas (Fig. 3). We did not observe MUC13 expression in squamous cell carcinoma components of the five adenosquamous cell carcinomas examined. Expression levels of MUC13 in carcinoma tissues were 
A
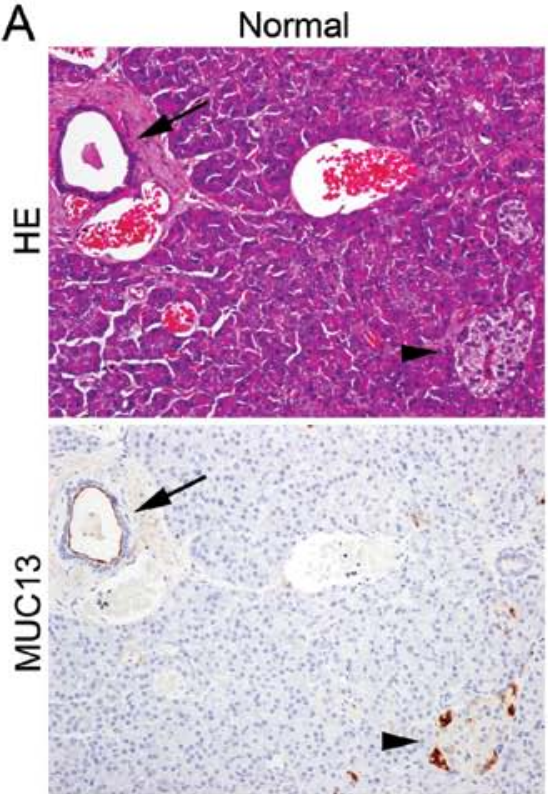

Por

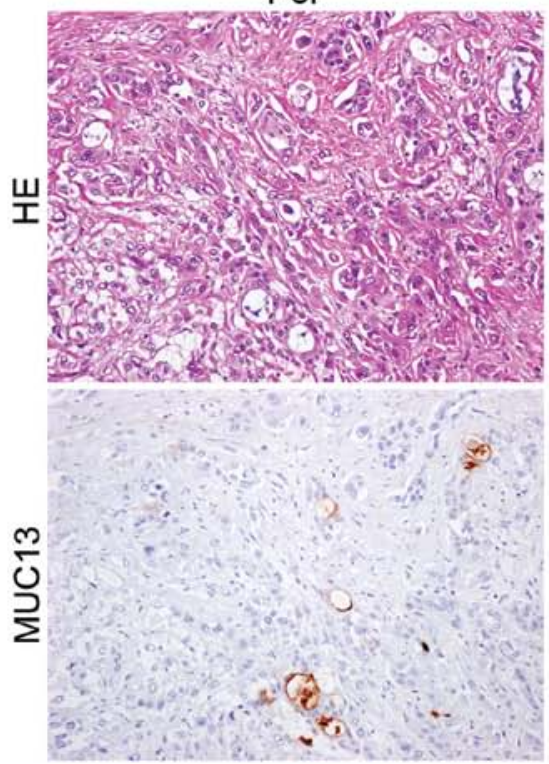

Wel
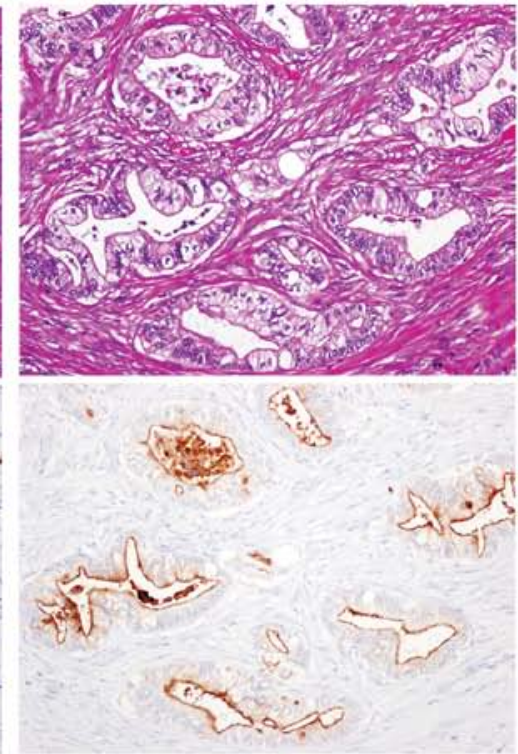

AS

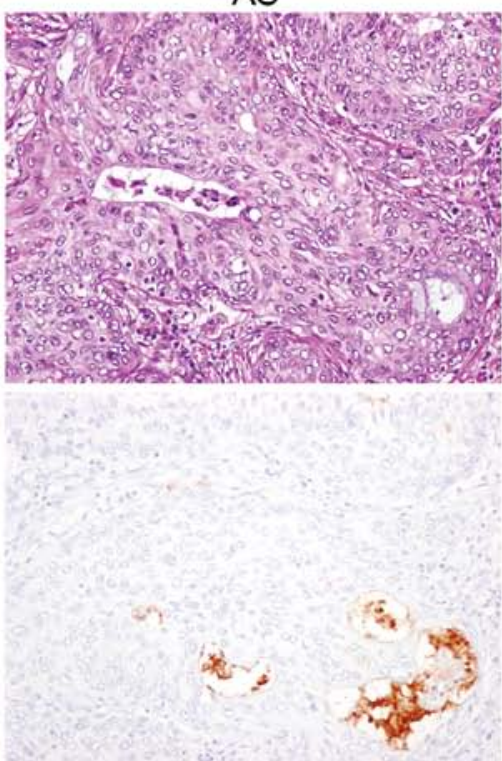

B

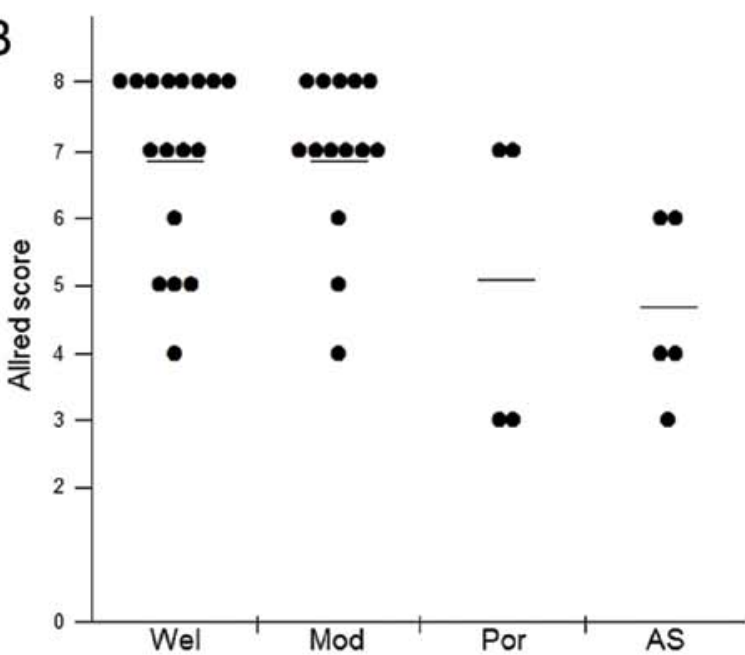

Figure 3. MUC13 is expressed in pancreatic cancer cells and normal ductal cells. (A) Representative images of MUC13 immunohistochemistry in the normal pancreas, well differentiated ductal adenocarcinoma, poorly differentiated ductal adenocarcinoma and adenosquamous cell carcinoma. Top panels, hematoxylin-eosin (HE) staining. Bottom panels, immunohistochemical staining for MUC13. The arrow $(\rightarrow)$ indicates pancreatic ducts. The arrowhead $(\bullet)$ shows an islet of Langerhans. Original magnification, x200. (B) Distribution of Allred scores for MUC13 expression among cancers of four histological subtypes. Wel, well differentiated ductal adenocarcinoma; mod, moderately differentiated ductal adenocarcinoma; por, poorly differentiated ductal adenocarcinoma; AS, adenosquamous cell carcinoma. 


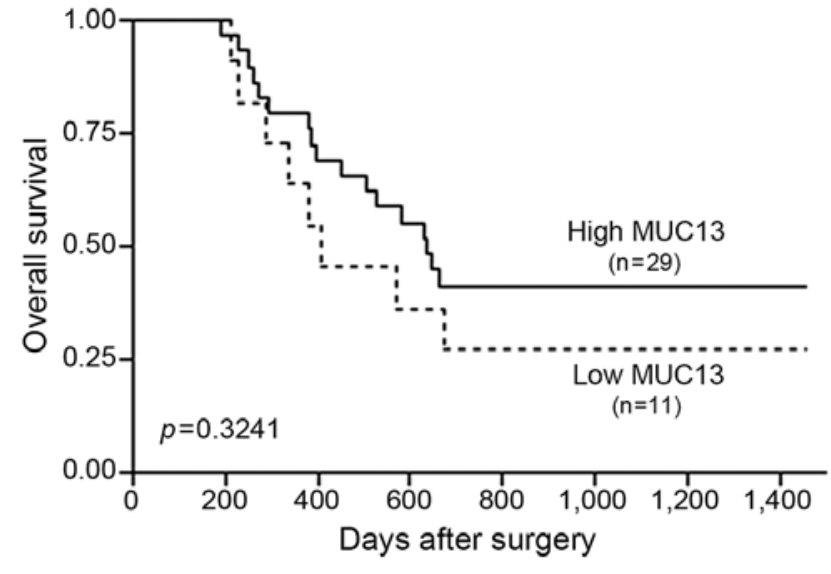

Figure 4. Low expression levels of MUC13 weakly correlated with decreased overall survival. Kaplan-Meier overall survival estimates for patients whose tumors contained low levels of MUC13 ( $\mathrm{n}=11$, Allred score $\leq 5)$ or high levels of MUC13 ( $n=29$, Allred score $\geq 6$ ). We determined $p$-values using the log-rank test.

slightly, but significantly higher than those in normal tissues (tumor vs. normal: $6.4 \pm 1.6$ vs. $5.6 \pm 0.9, \mathrm{p}=0.0023$ ).

Pancreatic carcinomas with low expression levels of MUC13 were associated with poor outcome. We examined if expression levels of MUC13 affected clinical outcome in patients with pancreatic cancers. Pancreatic carcinomas with lower MUC13 expression levels (Allred score $\leq 5, n=11$ ) were weakly associated with unfavorable outcomes compared with cancers exhibiting higher expression levels of MUC13 (Allred score $\geq 6, n=29$ ), however this difference was not statistically significant ( $\mathrm{p}=0.3241)$ (Fig. 4).

\section{Discussion}

We have developed a new anti-MUC13 mAb TCC56 to be internalized by cells using DT3C-based screening. Increased expression of MUC13 is reported for several carcinomas, including pancreatic cancer, therefore we hypothesized that MUC13 is a likely potential target molecule for cancer therapy (14-21). If an ADC comprising mAb TCC56 and a potent anticancer drug was produced, the new ADC could be effective for several cancers expressing MUC13. We have also demonstrated that all 40 pancreatic cancer tissues examined in this study expressed MUC13 at least partially, and that the expression levels of MUC13 in adenocarcinoma cells were significantly greater than those in normal ductal cells. Our findings suggest MUC13 is a candidate target molecule for treatment with ADCs. Our immunohistochemistry results revealed that well or moderately differentiated adenocarcinomas $(n=31)$ expressed MUC13 to greater extent than in poorly differentiated adenocarcinomas or adenosquamous cell carcinomas $(n=9)$. The MUC13 expression patterns observed suggest that an anti-MUC13 mAb-based drug could be applied to a wide range of pancreatic cancers. Cancers with low MUC13 expression levels appear to be somewhat more aggressive than those with high MUC13 expression levels led us to surmise that MUC13 did not play a critical role in the survival of pancreatic cancer cells. We attempted to repress MUC13 expression in
TCC-PAN2 cells using RNA interference techniques, however MUC13 expression was barely affected by the MUC13 short interfering RNA molecules we used, possibly because of very low transfection efficiencies (data not shown).

We postulated that MUC13 could not be targeted by ADCs, including anti-MUC13 mAbs, as its localization was confined to the apical membrane of cells. However, an ADC targeting an apical antigen has been developed previously and exhibited evidence of antitumor activity in phase I/II clinical trials involving patients with non-small cell lung cancers or ovarian cancers $(25,26)$. The apical antigen targeted is a $\mathrm{pH}$-sensitive sodium-dependent phosphate transporter encoded by the SLC34A2 gene, which is also known as NaPi2b (27,28). These data raise the possibility that ADCs, including anti-MUC13 mAb TCC56, could affect cases where MUC13 is clearly expressed in cancer cells.

In conclusion, we have developed a new anti-MUC13 mAb that could be efficiently internalized by cells. We have also demonstrated that MUC13 expression levels in pancreatic cancer tissues were higher than those in normal tissues, and that well or moderately differentiated ductal adenocarcinomas clearly expressed the protein. Our combined results suggest that MUC13 is a target molecule for pancreatic cancer treatment. ADCs, including anti-MUC13 mAbs, are promising anticancer agents that could alleviate the adverse effects of various chemotherapies.

\section{Acknowledgements}

We are grateful to Dr Hirofumi Hamada (former professor at Sapporo Medical University) for developing a sensitive screening method for selecting antibodies to be internalized by cells. We also thank Drs Mami Yamaguchi and Michitoshi Kimura (Sapporo Medical University School of Medicine) for assistance with immunohistochemistry.

\section{References}

1. Ryan DP, Hong TS and Bardeesy N: Pancreatic adenocarcinoma. N Engl J Med 371: 1039-1049, 2014.

2. Rossi ML, Rehman AA and Gondi CS: Therapeutic options for the management of pancreatic cancer. World J Gastroenterol 20: 11142-11159, 2014.

3. Michael $\mathrm{M}$ and Moore M: Clinical experience with gemcitabine in pancreatic carcinoma. Oncology (Williston Park) 11: 1615-1622, 1997.

4. Kindler HL: A new direction for pancreatic cncer treatment: FOLFIRINOX in context. Am Soc Clin Oncol Educ Book 2012: 232-237, 2012.

5. Von Hoff DD, Ervin T, Arena FP, et al: Increased survival in pancreatic cancer with nab-paclitaxel plus gemcitabine. N Engl J Med 369: 1691-1703, 2013.

6. Conroy T, Desseigne F, Ychou M, et al: FOLFIRINOX versus gemcitabine for metastatic pancreatic cancer. N Engl J Med 364: 1817-1825, 2011.

7. Panowksi S, Bhakta S, Raab H, Polakis P and Junutula JR: Sitespecific antibody drug conjugates for cancer therapy. MAbs 6 : 34-45, 2014.

8. Yamaguchi M, Nishii Y, Nakamura K, Aoki H, Hirai S, Uchida H, Sakuma Y and Hamada H: Development of a sensitive screening method for selecting monoclonal antibodies to be internalized by cells. Biochem Biophys Res Commun 454: 600-603, 2014.

9. Hollingsworth MA and Swanson BJ: Mucins in cancer: protection and control of the cell surface. Nat Rev Cancer 4: 45-60, 2004.

10. Kufe DW: Mucins in cancer: function, prognosis and therapy. Nat Rev Cancer 9: 874-885, 2009. 
11. Jonckheere N, Skrypek N and Van Seuningen I: Mucins and pancreatic cancer. Cancers 2: 1794-1812, 2010.

12. Kaur S, Kumar S, Momi N, Sasson AR and Batra SK: Mucins in pancreatic cancer and its microenvironment. Nat Rev Gastroenterol Hepatol 10: 607-620, 2013.

13. Williams SJ, Wreschner DH, Tran M, Eyre HJ, Sutherland GR and McGuckin MA: MUC13, a novel human cell surface mucin expressed by epithelial and hemopoietic cells. J Biol Chem 276 18327-18336, 2001.

14. Shimamura T, Ito H, Shibahara J, et al: Overexpression of MUC13 is associated with intestinal-type gastric cancer. Cancer Sci 96: 265-273, 2005.

15. Walsh MD, Young JP, Leggett BA, Williams SH, Jass JR and McGuckin MA: The MUC13 cell surface mucin is highly expressed by human colorectal carcinomas. Hum Pathol 38 : 883-892, 2007.

16. Chauhan SC, Vannatta K, Ebeling MC, et al: Expression and functions of transmembrane mucin MUC13 in ovarian cancer. Cancer Res 69: 765-774, 2009.

17. Maher DM, Gupta BK, Nagata S, Jaggi M and Chauhan SC: Mucin 13: structure, function, and potential roles in cancer pathogenesis. Mol Cancer Res 9: 531-537, 2011

18. Chauhan SC, Ebeling MC, Maher DM, Koch MD, Watanabe A, Aburatani H, Lio Y and Jaggi M: MUC13 mucin augments pancreatic tumorigenesis. Mol Cancer Ther 11: 24-33, 2012.

19. Gupta BK, Maher DM, Ebeling MC, et al: Increased expression and aberrant localization of mucin 13 in metastatic colon cancer. J Histochem Cytochem 60: 822-831, 2012.

20. Khan S, Ebeling MC, Zaman MS, et al: MicroRNA-145 targets MUC13 and suppresses growth and invasion of pancreatic cancer. Oncotarget 5: 7599-7609, 2014.
21. Gupta BK, Maher DM, Ebeling MC, Stephenson PD, Puumala SE, Koch MR, Aburatani H, Jaggi M and Chauhan SC: Functions and regulation of MUC13 mucin in colon cancer cells. J Gastroenterol 49: 1378-1391, 2014.

22. Suzuki K, Nakamura K, Kato K, et al: Exploration of target molecules for prostate cancer gene therapy. Prostate 67: 1163-1173, 2007.

23. Ishii K, Nakamura K, Kawaguchi S, et al: Selective gene transfer into neurons via $\mathrm{Na}, \mathrm{K}-\mathrm{ATP}$ ase beta1. Targeting gene transfer with monoclonal antibody and adenovirus vector. J Gene Med 10: 597-609, 2008.

24. Allred DC, Harvey JM, Berardo M and Clark GM: Prognostic and predictive factors in breast cancer by immunohistochemical analysis. Mod Pathol 11: 155-168, 1998.

25. Burris HA, Gordon MS, Gerber DE, et al: A phase I study of DNIB0600A, an antibody-drug-conjugate (ADC) targeting $\mathrm{NaPi} 2 \mathrm{~b}$, in patients (pts) with non-small cell lung cancer or platinum-resistant ovarian cancer (OC). J Clin Oncol 32 (Suppl) 2504, 2014

26. Ritter G, Yin B, Murray A, et al: Membrane transporter NaPi2b (SCL34A1) epitope for antibody therapy, antibodies directed thereto, and target for cancer therapy. US8603474. Issued December 10, 2013.

27. Murer H, Forster I and Biber J: The sodium phosphate cotransporter family SLC34. Pflugers Arch 447: 763-767, 2004.

28. Filonenko V, Gout T, Usenko VS, Lyzogubov VV, Shyian M and Kiyamova R: Immunohistochemical analysis of NaPi2b PROTEIN (MX35 antigen) expression and subcellular localization in human normal and cancer tissues. Exp Oncol 33: 157-161, 2011. 